\title{
Estrogen Activity Induction
}

National Cancer Institute

\section{Source}

National Cancer Institute. Estrogen Activity Induction. NCI Thesaurus. Code C41525.

Estrogen Activity Induction involves initiation of the biological function of biochemical substances (estrogens) secreted by the ovaries that have a specific regulatory effect on the activity of cells involved in development and maintenance of secondary female sex characteristics and control of the cyclical changes in the reproductive cycle, as well as pregnancy maintenance and an anabolic effect on protein metabolism and water retention. 